\title{
What controls filament thinning in uniaxial extension?
}

\author{
Walter Oswald ${ }^{\dagger}$, Steffen M. Recktenwald ${ }^{\dagger}$ and Norbert Willenbacher* \\ Institute for Mechanical Process Engineering and Mechanics, Karlsruhe Institute of Technology (KIT), \\ Karlsruhe 76131, Germany
}

\begin{abstract}
Breakup of fluid threads is omnipresent in nature and highly relevant for technical processes such as atomization, printing, coating, or spinning. We discuss how to control the filament lifetime of shear-thinning, viscoelastic fluids during uniaxial extension without affecting their shear viscosity. Two commercial acrylic thickeners differing with respect to the co-polymerized hydrophobic monomers, and hence different hydrophobic intra- and intermolecular association, are mixed to obtain aqueous solutions with similar shear viscosity. The elongational relaxation time as determined by capillary breakup extensional rheometry, however, varies by almost two orders of magnitude. Filament lifetime of these solutions can be varied upon adding non-Brownian, plate-shaped particles, again without affecting shear viscosity. A trace amount of particles increases elongational relaxation time by about a factor of four. Car bodies are usually coated using highspeed rotary bell atomizers guaranteeing high transfer efficiency and high-quality appearance. We use the solutions and suspensions described above to investigate the effect of extensional viscosity on ligament formation at the bell edge as a decisive intermediate step prior to droplet formation. High-speed imaging reveals a logarithmic scaling of ligament length with extensional relaxation time for pure thickener solutions. In contrast, ligament length monotonically decreases with increasing particle concentration, i.e. extensional viscosity. Plate-shaped particles obviously act as defects promoting ligament breakup. Extended filament lifetimes are commonly observed during atomization and spraying as a consequence of fluid viscoelasticity. On the other hand, low viscosity fluid threads rupture quickly. Here, we demonstrate that a unique banding instability during extension of a low viscosity surfactant solution with no measurable elasticity leads to extremely long filament lifetimes and to the formation of remarkably long threads. Combining filament stretching and particle image velocimetry we found an unexpected heterogeneous, banded flow in opposing directions. This phenomenon is not limited to surfactant solutions but can also occur in biopolymer solutions, thus broadening the view on instabilities in complex elongational flows.
\end{abstract}

Keywords: filament thinning, uniaxial extension, rotary bell atomization, flow instability

\section{Introduction}

The response of complex fluids to elongational deformation and flow is an intriguing phenomenon and a technological challenge. Drop deformation and filament breakup are dynamical processes ubiquitous in nature and a broad variety of technical processes such as atomization and spraying, fiber spinning or printing and coating operations include complex flow fields with strong elongational components. The elongational viscosity of nonNewtonian fluids generally depends on strain rate and total deformation as well as the kinematics of flow; often a steady state is hardly accessible. Measuring the elongational viscosity of low viscosity fluids is still challenging. Although converging channel and stagnation flows or filament stretching techniques are employed, limitations

\# This paper is based on an invited lecture presented by the corresponding author at the 30th Anniversary Symposium of the Korean Society of Rheology (The 18th International Symposium on Applied Rheology (ISAR)), held on May 21 24, 2019, Seoul.

'These authors contributed equally.

*Corresponding author; E mail: norbert.willenbacher@kit.edu occur with respect to the uniformity of the flow field or the correlation of measured stress signals to strain rate and total strain.

During the last two decades capillary breakup elongational rheometry (CaBER) has been widely used to characterize a wide variety of soft complex fluids from dilute, weakly viscoelastic polymer solutions (Rodd et al., 2005) to highly elastic yield stress fluids (Martinie et al., 2013).

Its captivating simplicity of application has stimulated numerous investigations aiming at a fundamental understanding of elongational flow behavior of low viscosity fluids as well as on correlations between capillary thinning of such fluids and their behavior in technical processes. However, it has to be kept in mind, that the strain rate cannot be pre-selected or varied but is set by the fluid itself. Furthermore, the true elongational stress is generally not accessible, only the so-called tilted CaBER allows for a calculation of the axial force using filament thinning based on the gravitational deflection of the slender threads (Sachsenheimer et al., 2012).

Based on CaBER experiments we focus on two phenomena here. First, we discuss how elongational viscosity 
affects high-speed rotary bell atomization, the global standard technology in automotive coating. To that end, we have investigated two series of highly shear-thinning model systems with a broad variation in elongational relaxation time but essentially constant shear viscosity in a broad shear rate range relevant for processing and atomization. This research highlights how differently the deformability or flexibility of dissolved polymeric thickeners and the addition of plate-shaped particles affect ligament detachment and droplet formation even if they have a similar effect on elongational flow resistance (Oswald and Willenbacher, 2019; Oswald et al., 2019).

Second, we discuss how the filament lifetime of low viscosity fluids in CaBER experiments or other uniaxial extensional flows is dramatically extended due to the onset of a flow instability (Recktenwald et al., 2019). Particle image velocimetry (PIV) experiments combined with horizontal filament stretching directly show a heterogeneous flow field in dilute surfactant systems as well as biopolymer solutions. This broadens our view on the occurrence of flow stabilities in different flow kinematics but also highlights the limitations of extensional viscosity and relaxation time determination simply from filament thinning kinetics in CaBER experiments.

\section{Experimental}

\subsection{Materials}

Aqueous solutions of Sterocoll ${ }^{\circledR}$ HT and Sterocoll ${ }^{\circledR}$ XT (BASF SE, Ludwigshafen, Germany) mixtures were prepared as described in Oswald and Willenbacher (2019). These thickeners are copolymers including ethyl acrylate and carboxylic acids. The random occurrence of hydrophobic segments along the polymer backbone causes interas well as intramolecular hydrophobic associations which give rise to a unique flow behaviour and thickening efficiency (Kheirandish et al., 2009). Sterocoll ${ }^{\circledR}$ HT includes a higher fraction of hydrophobic monomer than for Sterocoll $^{\circledR}$ XT. Accordingly, the density of intra- and inter-

Table 1. Composition of mixed aqueous Sterocoll ${ }^{\circledR}$ solutions $(\mathrm{pH}$ $=8$ ). Glass flakes of the type Luxan CFX C001 (Eckart GmbH, Hartenstein, Germany) were dispersed into solution S3 at differ ent volume fractions $\varphi_{F}=0.6,1.3,2.0$, and $3.5 \mathrm{vol} . \%$. According to the provider the average diameter of these flakes is in the range of $2535 \mathrm{~mm}$ and the aspect ratio is $\mathrm{AR} \approx 25$.

\begin{tabular}{ccc}
\hline \hline Label & $\begin{array}{c}c\left(\text { Sterocoll }^{\circledR} \mathrm{HT}\right) \\
{[\text { wt. } \%]}\end{array}$ & $\begin{array}{c}\left.c \text { (Sterocoll }^{\circledR} \mathrm{XT}\right) \\
{[\mathrm{wt.} \%]}\end{array}$ \\
\hline S1 & 2.3 & 0 \\
S2 & 2.0 & 0.15 \\
S3 & 1.8 & 0.22 \\
S4 & 1.7 & 0.3 \\
S5 & 1.6 & 0.4 \\
\hline
\end{tabular}

molecular hydrophobic associations is higher and these molecules are stiffer and less flexible in solution than Sterocoll $^{\circledR}$ XT. Composition of the investigated solutions is listed in Table 1.

Solutions of the anionic surfactant hexadecyltrimethylammonium bromide (CTAB) and the salt sodium salicylate $(\mathrm{NaSal})$ were prepared at a surfactant concentration of $10 \mathrm{mM}$, covering a broad range of salt/surfactant ratio $0.4<R<20$. At low salt content, solutions consist of small, rod-like micelles and exhibit a zero-shear viscosity close to that of water in steady shear experiments, followed by a shear-thickening transition due to the formation of shear-induced structures (SIS) (Rehage and Hoffmann, 1982). On the other hand, solutions with a higher amount of salt consist of long, flexible wormlike micelles (WLM) that can entangle, thus promote viscoelasticity. These solutions show a zero-shear viscosity, orders of magnitudes higher than that of water, followed by shear-thinning behavior.

Further, we studied the uniaxial flow behavior of an aqueous 0.14 wt.\% hagfish mucin solution, provided by the IFNH Food Process Engineering Group of ETH Zurich. In CaBER experiments, hagfish solutions were shown to exhibit delayed filament breakup and pronounced strain hardening (Böni et al., 2016).

\subsection{Methods}

The steady shear viscosity of mixed Sterocoll ${ }^{\circledR}$ solutions at shear rates $300<\dot{\gamma}<300,000 \mathrm{~s}^{-1}$ was determined using a customized piston driven capillary rheometer equipped with a circular die (radius $r_{\text {cap }}=0.25 \mathrm{~mm}$, length $L=40$ $\mathrm{mm}$ ). The measuring temperature was $T=25^{\circ} \mathrm{C}$.

Uniaxial elongational flow behavior was probed using a capillary breakup elongational rheometer (CaBER-1 Thermo Scientific, Germany). We chose circular plates with a diameter of $D_{0}=6 \mathrm{~mm}$, the initial and final displacement was $h_{i}=1.5 \mathrm{~mm}$ and $h_{f}=6 \mathrm{~mm}$ for the surfactant as well as biopolymer solutions, for the Sterocoll ${ }^{\circledR}$ solutions we chose $h_{i}=2 \mathrm{~mm}$ and $h_{f}=10 \mathrm{~mm}$. The strike time was always $t_{s}=40 \mathrm{~ms}$. The filament diameter was recorded over time and the filament lifetime $t_{f i l}$ was determined when the liquid thread broke. Furthermore, we determined the filament lifetime ratio $t_{f i l} / t_{f i l, N}$ by calculating the theoretical filament lifetime $t_{f i l, N}$ from the zero-shear viscosity of the surfactant solutions, assuming Newtonian flow behavior (Sachsenheimer et al., 2014). The elongational relaxation time $\lambda_{e}$ of the Sterocoll ${ }^{\circledR}$ solutions was calculated from the change in filament diameter $D(t)$ in the elasto-capillary regime, in which $D(t)$ decays exponentially (Entov and Hinch, 1997):

$$
\lambda e=\begin{gathered}
D(t) \\
3 d D(t) / d t
\end{gathered} .
$$

The velocity field inside a thinning $10 \mathrm{mM} \mathrm{CTAB/}$ 
NaSal, $R=0.5$ surfactant filament was determined using a filament stretching device mounted on an inverted fluorescence microscope AxioObserver D (Carl Zeiss AG, Germany) in combination with particle image velocimetry (PIV) (Recktenwald et al., 2019). The sample was seeded with $0.01 \mathrm{wt} . \%$ of fluorescent polystyrene beads $\left(d_{p}=1\right.$ $\mu \mathrm{m}$, Banglabs Inc., USA) and stretched using plates with $D_{0}=4 \mathrm{~mm}, h_{i}=1 \mathrm{~mm}$ and $h_{f}=4 \mathrm{~mm}$, and a strike time of $t_{s}=1 \mathrm{~s}$. Images in different filament layers of the center part of the surfactant filament were captured using a long travel range objective scanner ( $\mathrm{P}-\mathrm{a}$ 725.4CD, Physik Instrumente $\mathrm{GmbH} \& \mathrm{Co}$. KG, Germany), a high-speed camera (Zyla X camera, Andor Technology, Ireland) and a 20x long working distance lens (Nikon LU-Plan, NA = $0.4)$. The velocity components inside the thinning filament were obtained using an open source PIV software (Thielicke and Stamhuis, 2014).

Atomization of thickener solutions with and without glass flakes was performed using the high-speed rotary bell atomizer EcoBell2 (Dürr AG, Germany). The bell cup has a contour angle of $62^{\circ}$ and diameter of $65 \mathrm{~mm}$ including serrations at the bell edge with a width of $0.12 \mathrm{~mm}$. The setup was placed in an air-conditioned spray cabin ( $T$ $=25^{\circ} \mathrm{C}$, relative humidity $=65 \%$ ). Liquid flow rate and rotational speed were varied between $\dot{V}=200-350 \mathrm{ml} /$ min and $n=10-50 \mathrm{krpm}$, respectively. The airflow rate was kept constant at $340 \mathrm{Nl} / \mathrm{min}$. Formation and disintegration of ligaments near the bell edge were recorded at a resolution of $768 \times 1024$ pixels and a frame rate of 25,000 fps using a Fastcam SA-Z (Photron Tokyo, Japan) highspeed camera. Ligament length were determined using a self-written image analysis MATLAB code as described in Oswald et al. (2019). In each atomization experiment about 1,000-2,000 images were analyzed corresponding to approximately 20,000 ligaments.

\section{Results and Discussion}

Mixtures of the commercial alkali-swellable-emulsion

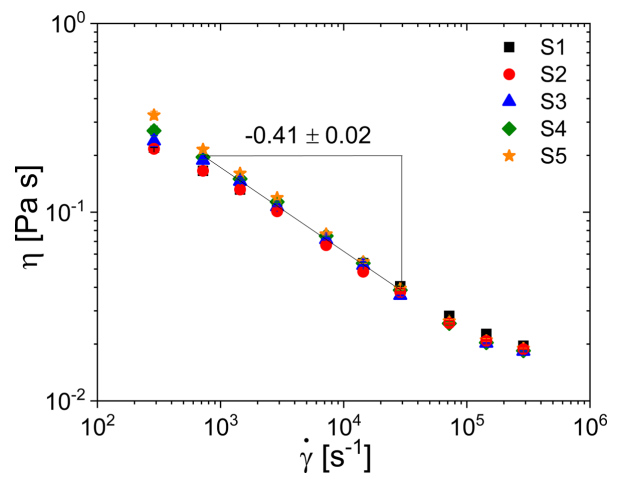

Fig. 1. (Color online) Shear viscosity $\eta$ versus shear rate $\dot{\gamma}$ for mixed Sterocoll ${ }^{\circledR}$ solutions S1 S5 as determined from capillary rheometry. type acrylate thickeners Sterocoll ${ }^{\circledR}$ XT and Sterocoll ${ }^{\circledR}$ HT, as summarized in Table 1, were used as model system for investigating the relevance of elongational flow behavior on high-speed rotary bell atomization.

Figure 1 clearly shows that these solutions are highly shear-thinning and the viscosity $\eta$ decreases with increasing shear rate $\dot{\gamma}$ according to a power law $\eta \sim \dot{\gamma}^{n-1}$ with $n=0.59 \pm 0.02$. The absolute viscosity values are essentially independent of sample composition in the whole shear rate range investigated here. In contrast, the elongational relaxation time $\lambda_{e}$, determined from the decay of the $D(t)$ curves in the elasto-capillary thinning regime during CaBER experiments, strongly increases with increasing fraction of Sterocoll ${ }^{\circledR}$ XT in these mixtures (Fig. 2). We attribute this to the higher flexibility of this polymer compared to the Sterocoll ${ }^{\circledR}$ HT as explained in detail elsewhere (Oswald and Willenbacher, 2019).

Based on mixture S3 we have prepared a second series of samples including different fractions of suspended glass flakes. Up to $3.5 \mathrm{vol} \%$ of these plate-shaped particles were suspended in solution $\mathrm{S} 3$ and as expected this does not alter the shear viscosity (Oswald and Willenbacher, 2019). As can be seen from Fig. 3a, the addition of the glass flakes, however, results in a strong increase of elongational relaxation time from $\lambda_{e}=31 \mathrm{~ms}$ for $\mathrm{S} 3$ to $\lambda_{e}=$ $131 \mathrm{~ms}$ for the suspension including 3.5 vol.\% glass flakes. These values are determined in the so-called elastocapillary thinning regime and according to Fig. 3a the filament diameter $D(t)$ is much larger than the flake dimensions in this regime. To ensure that $\lambda_{e}$ data represent the behavior of the suspensions in uniform uniaxial extension we added tracer particles (diameter $d_{p}=1 \mu \mathrm{m}$ ) to visualize the flow. Fluorescent microscopy images (Fig. 3b) subsequently taken at time intervals $\Delta t=280 \mathrm{~ms}$ confirm, that the glass flakes with a density of $\rho=2600 \mathrm{~kg} \mathrm{~m}^{-3}$ flow at

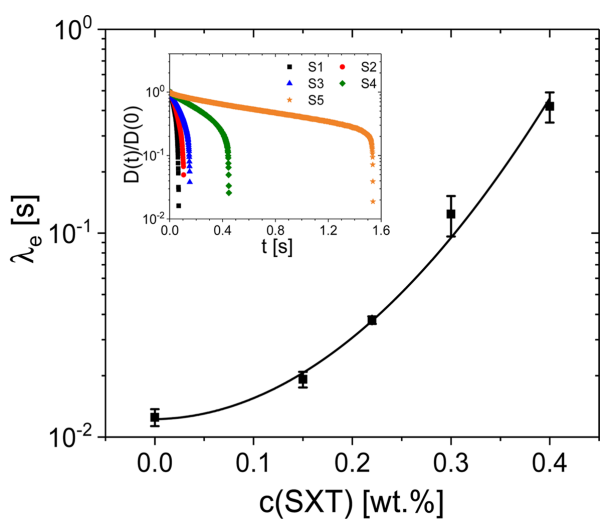

Fig. 2. (Color online) Elongational relaxation times $\lambda_{e}$ vs. con centration of Sterocoll ${ }^{\circledR}$ XT c(SXT) for mixed Sterocoll ${ }^{\circledR}$ solu tions S1 S5. Inset: Corresponding filament diameter $D(t)$ normalized by its initial value $D(t=0)$ right after the piston has reached its final position $h_{f}$ vs. filament thinning time. The line is to guide the eye. 

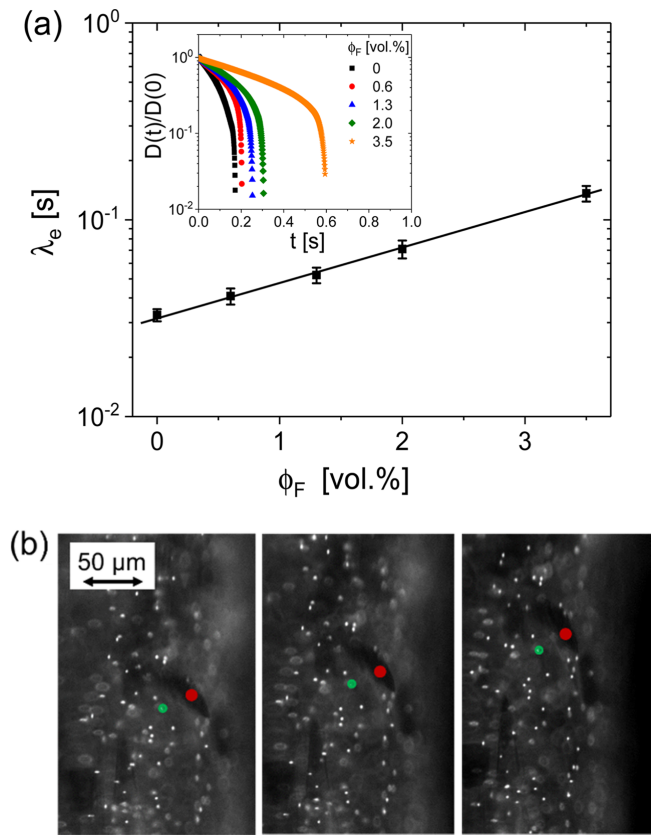

Fig. 3. (Color online) CaBER experiments on thickener solution S3 including glass flakes Luxan CFX C001 (average diameter: $2535 \mathrm{~mm}$, aspect ratio is $\mathrm{AR} \approx 25$ ). (a) Elongational relaxation time $\lambda_{e}$ vs. particle concentration $\phi_{F}$ for solution S3 including dif ferent concentrations of glass flakes. Inset: Corresponding fila ment diameter $D(t)$ normalized by its initial value $D(0)$ right after the piston has reached its final position $h_{f}$ vs. filament thinning time $t$. (b) Comparing flow of glass flakes (red dot) and tracer particles (green dot) in subsequent images taken at time intervals $\Delta t=280 \mathrm{~ms}$ during horizontal filament stretching of a mixed Ste rocoll $^{\circledR}$ solution including $1 \mathrm{wt} \% \%$ Sterocoll $^{\circledR}$ HT and $1 \mathrm{wt} \% \%$ Ste rocoll $^{\circledR}$ XT.

the same velocity as the tracer particles showing a density of $\rho=1040 \mathrm{~kg} \mathrm{~m}^{-3}$ similar to the thickener solution, i.e. the extensional flow seems not to be disturbed by inertia. A similarly strong effect of anisotropic particles on extensional flow resistance of dilute suspensions has been reported earlier for plate-shaped fillers dispersed in a polymer melt by Utracki and Lara (1984), and also for sus- pensions of rod-like particles in Newtonian fluids by Weinberger and Goddard (1974).

Next, we discuss how these model systems behave in high-speed rotary bell atomization. This is the global standard coating technology in the automotive industry. Driven by centrifugal forces the coating flows from the center supply of a cup-shaped rotating bell to its edge. Slender ligaments form and detach at the rim and finally disintegrate in droplets coating the car body (see Fig. 4). Highspeed imaging combined with customized image analysis has been used to determine the length of the ligaments forming at the bell edge (Oswald et al., 2019). Ligament length $L_{\text {lig }}$ data for samples S1 to S5 obtained at volumetric flow rates $\dot{V}=200 \mathrm{ml} / \mathrm{min}$ and $350 \mathrm{ml} / \mathrm{min}$ are shown in Fig. 5a. In both cases rotational speed was $n=10 \mathrm{krpm}$, but similar results were obtained at other speeds. Obviously, $L_{\text {lig }}$ increases logarithmically with increasing $\lambda_{e}$. In both flow types the increasing fraction of flexible polymer seems to stabilize the fluid threads. Besides that, increasing volume flow rate leads to an increase of ligament length, since film thickness and hence, initial diameter of the ligaments is increased as well.

The situation is different for the solutions including glass flakes. In this case, ligament length $L_{\text {lig }}$ decreases with increasing $\lambda_{e}$ and this decay is independent of rotational speed $\mathrm{n}$ as can be seen in Fig. 5b. Elongational relaxation time $\lambda_{e}$ data are determined from thinning filaments with diameter $D(t)$ much larger than the glass flake dimensions and reflect the elongational flow resistance of the suspensions on a continuum mechanical level. The ligaments detaching from the bell edge during atomization, however, exhibit diameters similar to that of the glass flakes. Thus, we assume these flakes to act as predetermined breaking points and accordingly the ligament length decreases with increasing concentration of glass flakes. It should be noted, that the reduction in ligament length is much less pronounced and essentially independent of particle concentration when glass spheres are added. It seems that the sharp edges of the flakes promote

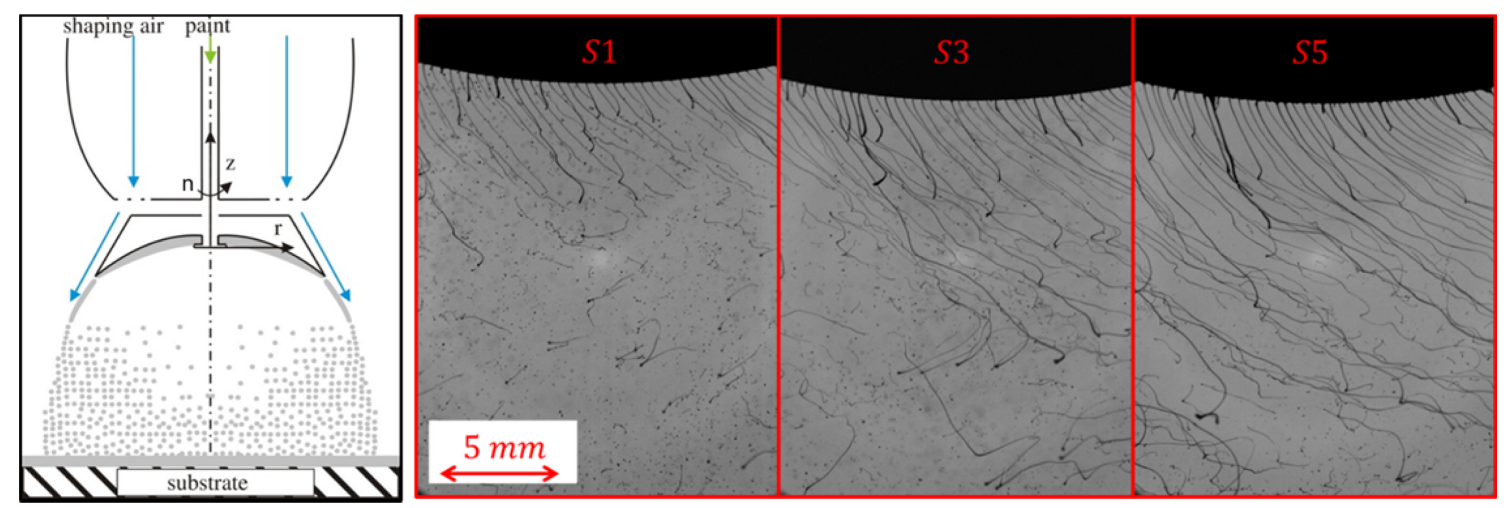

Fig. 4. (Color online) Scheme of atomization process in a high speed rotary bell atomizer (left) and high speed recordings of ligament formation at the bell edge for solutions $\mathrm{S} 1, \mathrm{~S} 3$, and S5 taken at rotational speed $n=20 \mathrm{krpm}$ and paint flow rate $\dot{V}=350 \mathrm{ml} / \mathrm{min}$ (right). 

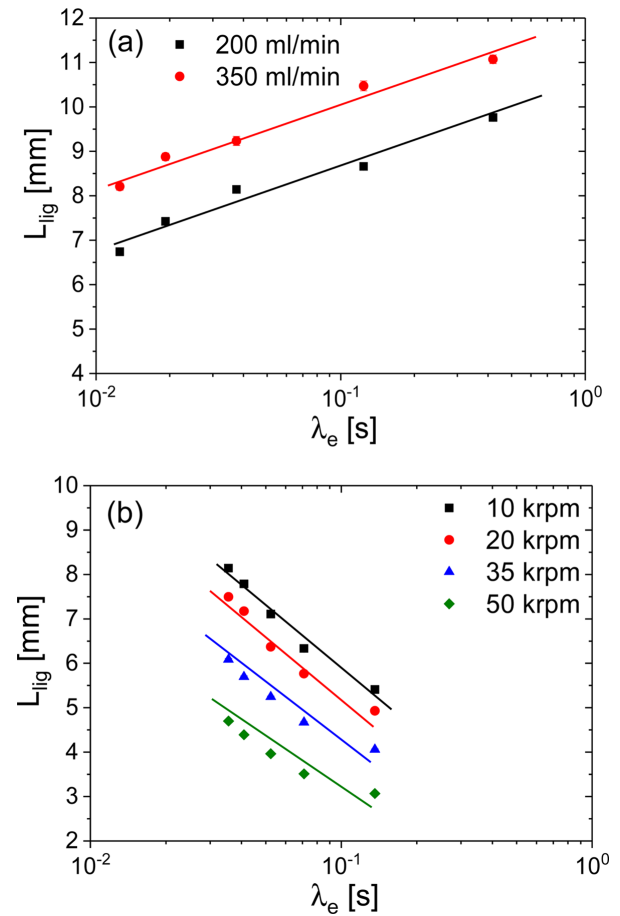

Fig. 5. (Color online) (a) Length of ligaments $L_{\text {lig }}$ vs. elongational relaxation time $\lambda_{e}$ for mixed Sterocoll ${ }^{\mathbb{B}}$ solutions S1 S5 obtained at rotational speed $n=10 \mathrm{krpm}$ and paint flow rate $\dot{V}=200 \mathrm{ml} /$ min and $350 \mathrm{ml} / \mathrm{min}$ and (b) solution S3 including different con centrations of glass flakes obtained at flow rate $\dot{V}=200 \mathrm{ml} / \mathrm{min}$ and different rotational speeds $n=10,20,35$, and $50 \mathrm{krpm}$.

ligament breakup and detachment. Remarkably, the length of the ligaments formed at the bell edge has no effect on the size of the droplets finally hitting the substrate to be coated, this is true for the pure solutions as well as for the systems including glass flakes (Oswald et al., 2019).

CaBER experiments have been widely used to study the elongational flow behavior of dilute and semi-dilute polymer solutions as well as surfactant solutions forming rodor wormlike micelles (Bhardwaj et al., 2007; Clasen, 2010; Sachsenheimer et al., 2014). Particularly the relationship between $\lambda_{e}$ and shear relaxation time $\lambda_{s}$ has been discussed intensively. For solutions of linear, flexible polymers $\lambda_{e} \leq \lambda_{s}$ is found, but $\lambda_{e} / \lambda_{s}$ monotonically decreases with polymer concentration (Sachsenheimer et al., 2014). Sterocoll ${ }^{\circledR}$ solutions with their strong inter- and intramolecular hydrophobic associations exhibit $\lambda_{e}<<\lambda_{s}$ (Kheirandish et al., 2009).

In contrast, dilute surfactant solutions at low salt/surfactant ratio $\mathrm{R}$ with no measurable viscoelasticity exhibit relaxation times and filament lifetimes orders of magnitude larger than expected from their low viscosity in steady shear experiments. In Fig. 6a this is shown for 10 $\mathrm{mM} \mathrm{CTAB} / \mathrm{NaSal}$ solutions, where we plot the filament lifetime ratio $t_{f i l} / t_{f i l, N}$ of the solutions as a function of the salt/surfactant ratio (Sachsenheimer et al., 2014). In fact,
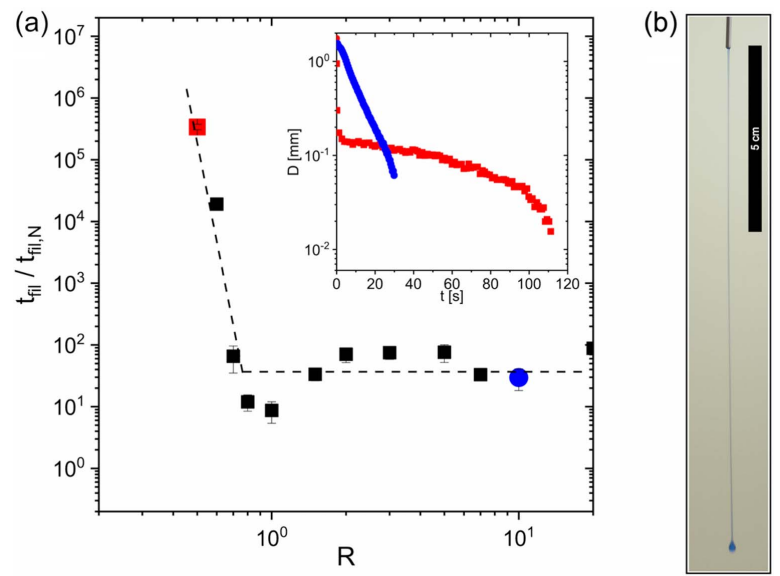

Fig. 6. (Color online) Macroscopic filament breakup of surfac tant solutions. (a) Filament lifetime ratio of $10 \mathrm{mM} \mathrm{CTAB/NaSal}$ solutions as a function of salt/surfactant ratio. Dashed lines are to guide the eye. The inset in (a) shows the diameter over time in CaBER experiments. The red squares and the blue circles cor respond to $R=0.5$ and $R=10$, respectively. (b) Formation of a remarkably long surfactant filament when a drop of a $10 \mathrm{mM}$ CTAB/NaSal, $R=0.5$ solution detaches from a needle. The black bar represents a length of $5 \mathrm{~cm}$.

the filament lifetime of the solution with $R=0.5$ is clearly longer than for the solution with $R=10$ (see inset in Fig. $6 \mathrm{a})$, despite its much lower zero shear viscosity $\left(\eta_{0}=\right.$ 0.003 Pas for $R=0.5$ and $\eta_{0}=3$ Pas for $R=10$ ). In dripping experiments, these low viscosity surfactant solutions form excessively long filaments. For the $10 \mathrm{mM} \mathrm{CTAB} /$ NaSal, $R=0.5$ solution, more than $10 \mathrm{~cm}$ in length are obtained as can be seen from Fig. 6b. Such unusual behavior was attributed to elongation-induced structure formation, i.e., growth and aggregation of micelles during extensional deformation (Sachsenheimer et al., 2014; Omidvar et al., 2018).

Recently, Recktenwald et al. (2019) revealed that these extremely long filament lifetimes observed in CaBER experiments on dilute surfactant solutions with low $R$ are a consequence of a flow instability induced during the initial step strain. Performing PIV experiments in horizontally stretched filaments using the setup shown in Fig. 7a, they could show that fluid bands with different velocities across the filament cross-section exist, transporting the fluid in both axial directions simultaneously and even from the end reservoirs back into the filament. Based on such experiments they established state diagrams indicating when such a heterogeneous flow inside the filament, a uniform flow during filament thinning, or even no filament formation occurs covering a broad surfactant concentration and $R$ range for four widely used surfactant systems. Complementary experiments in planar extension as well as in shear revealed that these surfactant solutions exhibit a flow instability characterized by a multivalued 
(a)

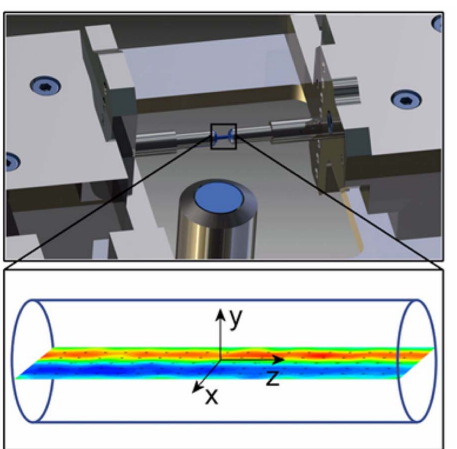

(c)

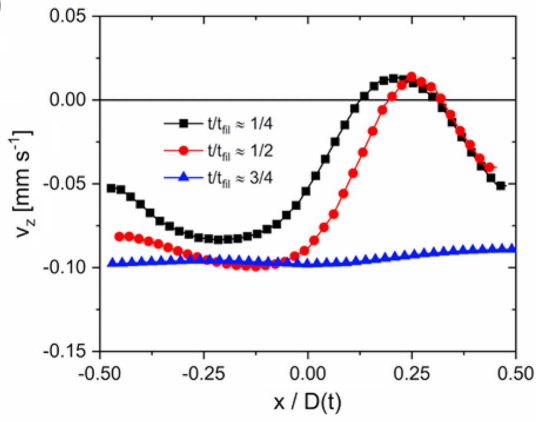

(b) $\quad \mathrm{v}_{\mathrm{z}}\left[\mathrm{mm} \mathrm{s}^{-1}\right]$

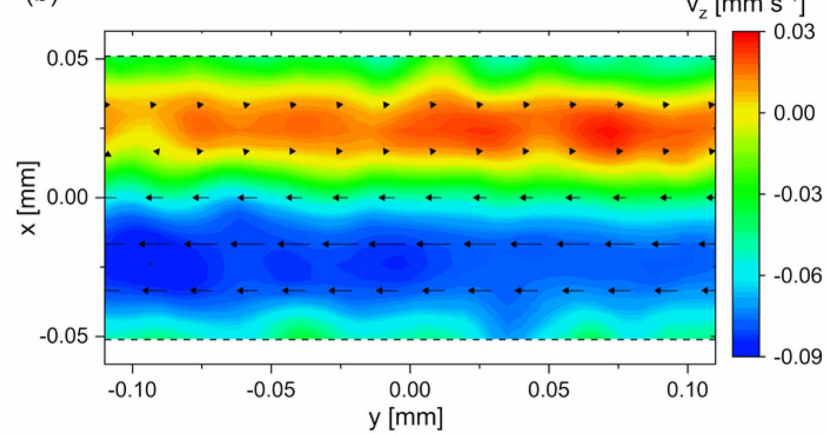

(d)

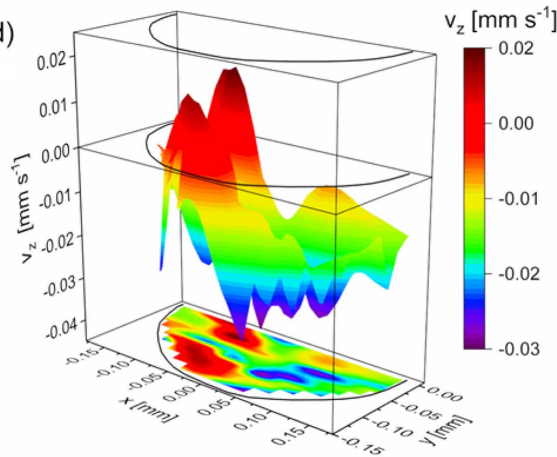

Fig. 7. (Color online) Microscopic flow behavior inside thinning surfactant filaments using a $10 \mathrm{mM} \mathrm{CTAB} / \mathrm{NaSal}, R=0.5$ solution. (a) Schematic representation of the device for horizontal filament stretching and PIV experiments, adapted from Recktenwald et al. (2019). (b) Velocity component in axial filament direction $v_{z}$ inside the midplane of the circular filament cross section. (c) $v_{z}$ over nor malized filament diameter for three times during filament thinning at $y=0$. (d) Three dimensional (3D) representation of $v_{z}$ in the lower half of the circular surfactant filament cross section. A projection of $v_{z}$ in the $x y$ plane is inserted at the bottom of the graph.

stress arising beyond a critical strain rate, irrespective of flow kinematics.

The heterogeneous, unstable flow in a $10 \mathrm{mM} \mathrm{CTAB} /$ NaSal solution, $R=0.5$ is illustrated in Fig. 7. The existence of flow bands moving at different velocity and even opposite direction is clearly seen in Fig. 7b. The velocity component in axial direction $v_{z}$ across the filament middle plane $(y=0)$ is shown in Fig. 7c for different times during filament thinning. This illustrates the temporal change of the velocity profile, i.e., the instability of the flow. Note, that all other velocity vector components except $v_{z}$ are negligibly small. Figure $7 \mathrm{~d}$ shows that the heterogeneous flow is not restricted to the middle plane of the filament but occurs in the whole filament cross-section and again it is obvious that fluid transport takes place in both axial directions.

Finally, we emphasize that such unexpectedly long filament lifetimes and flow instabilities in uniaxial extensions are not just a curiosity in dilute surfactant solutions. Figure 8 demonstrates that such phenomena are also present in biopolymer solutions. A $0.14 \mathrm{wt} . \%$ hagfish mucin solution has been studied exhibiting a filament lifetime of several seconds in CaBER experiments (Fig. 8a). Here, the flow inside the liquid thread is also heterogeneous and
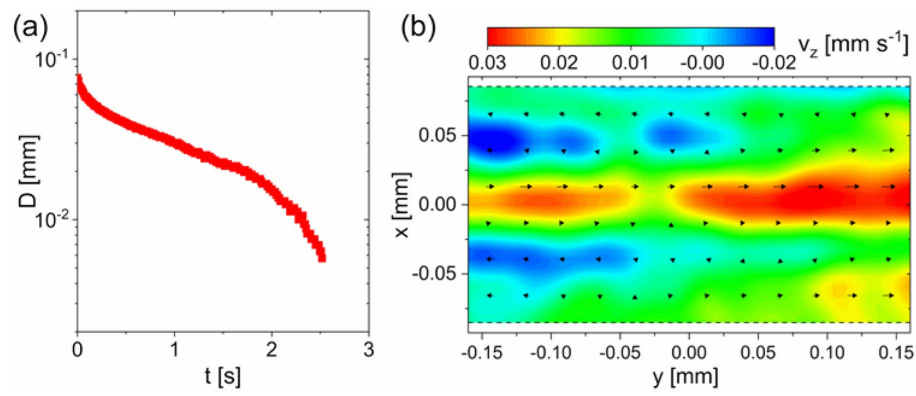

(c)

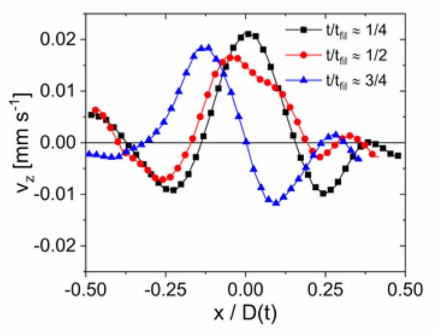

Fig. 8. (Color online) Uniaxial deformation of a 0.14 wt.\% hagfish mucin solution. (a) Diameter over time in CaBER experiment with $D_{0}=6 \mathrm{~mm}, h_{i}=1.5 \mathrm{~mm}$ and $h_{f}=7 \mathrm{~mm}$, and $t_{s}=40 \mathrm{~ms}$. (b) Velocity component in axial filament direction $v_{z}$ inside the midplane of the circular filament cross section, adapted from Recktenwald et al. (2019). (c) $v_{z}$ across the normalized filament diameter for three times during filament thinning at $y=-0.1 \mathrm{~mm}$. 
the PIV data presented in Fig. 8b shows several bands flowing at different absolute velocities in opposite axial directions within the filament middle plane. The temporal change in the velocity profile across the filament profile is illustrated in Fig. 8c showing $v_{z}$ versus normalized position $x / D(t)$ at different times during filament thinning.

\section{Conclusion}

Elongational relaxation time $\lambda_{e}$ in uniaxial filament thinning of complex, shear-thinning fluids including dissolved polymer molecules and anisotropic solid particles can be varied in a wide range depending on polymer flexibility and concentration of added particles. Here, we have demonstrated that these concepts can be used to control elongation relaxation time of acrylic thickener solutions closely resembling the shear flow behavior of commercial waterborne automotive coatings without affecting shear rheology in a wide processing and application relevant shear rate range. Corresponding solutions and suspensions of such thickener solutions have been used as model systems to study the effect of elongational flow resistance in highspeed rotary bell atomization, the global standard in automotive coating business. We investigated ligament formation at the bell edge as a decisive intermediate step prior to droplet formation. High-speed imaging revealed a logarithmic scaling of ligament length with extensional relaxation time for the pure thickener solutions. Dissolved flexible polymer chains stabilize both types of flows. In contrast, ligament length monotonically decreased with increasing particle concentration, i.e., extensional relaxation time. Plate-shaped particles obviously act as defects promoting ligament breakup.

Extended filament lifetimes in uniaxial elongational flows are commonly observed for strongly viscoelastic fluids. Here, we show how a low viscosity surfactant solution with no measurable elasticity can exhibit extremely long filament lifetimes in filament stretching experiments. Combining filament stretching and particle image velocimetry we found a heterogeneous, banded flow in opposing axial directions with time-dependent velocity profiles. Velocities vary in magnitude and sign across the filament cross-section and even flow reversal arises during filament thinning. This phenomenon not only occurs in dilute surfactant solutions at low salt content but also in biopolymer solutions. These findings broaden our view on instabilities occurring in complex flows with strong elongational components and set new limitations for the evaluation of extensional relaxation times from filament thinning experiments.

\section{Acknowledgements}

We appreciate financial support by BASF Coatings $\mathrm{GmbH}$ and the German Science Foundation DFG (grant number WI 3138/22-1).

\section{References}

Bhardwaj, A., E. Miller, and J.P. Rothstein, 2007, Filament stretch ing and capillary breakup extensional rheometry measurements of viscoelastic wormlike micelle solutions, J. Rheol. 51, 693719.

Böni, L., P. Fischer, L. Böcker, S. Kuster, and P.A. Rühs, 2016, Hagfish slime and mucin flow properties and their implications for defense, Sci. Rep. 6, 30371.

Clasen, C., 2010, Capillary breakup extensional rheometry of semi dilute polymer solutions, Korea Aust. Rheol. J. 5, 331338.

Entov, V.M. and E.J. Hinch, 1997, Effect of a spectrum of relax ation times on the capillary thinning of a filament of elastic liq uid, J. Non Newton Fluid Mech. 72, 3153.

Kheirandish, S., I. Gubaydullin, and N. Willenbacher, 2009, Shear and elongational flow behavior of acrylic thickener solu tions. Part II: Effect of gel content, Rheol. Acta 48, 397407.

Martinie, L., H. Buggisch, and N. Willenbacher, 2013, Apparent elongational yield stress of soft matter, J. Rheol. 57, 627646.

Omidvar, R., A. Dalili, A. Mir, and H. Mohammadigoushki, 2018, Exploring sensitivity of the extensional flow to wormlike micellar structure, J. Non Newton. Fluid Mech. 252, 4856.

Oswald, W., L. Gödeke, P.Ehrhard, and N. Willenbacher, 2019, Influence of the elongational flow resistance and pigmentation of coating fluids on high speed rotary bell atomization, At. Sprays, being reviewed.

Oswald, W. and N. Willenbacher, 2019, Controlling elongational flow behavior of low viscosity complex fluids at constant shear viscosity, Rheol. Acta 58, 687698.

Recktenwald, S.M., S.J. Haward, A.Q. Shen, and N. Willenbacher, 2019, Heterogeneous flow inside threads of low viscosity flu ids leads to anomalous long filament lifetimes, Sci. Rep. 9, 7110.

Rehage, H. and H. Hoffmann, 1982, Shear induced phase tran sitions in highly dilute aqueous detergent solutions, Rheol. Acta 21, 561563.

Rodd, L.E., T.P. Scott, J.J. Cooper White, and G.H. McKinley, 2005, Capillary break up rheometry of low viscosity elastic fluids, Appl. Rheol. 15, 1227.

Sachsenheimer, D., B. Hochstein, H. Buggisch, and N. Willen bacher, 2012, Determination of axial forces during the capil lary breakup of liquid filaments The tilted CaBER method, Rheol. Acta 51, 909923.

Sachsenheimer, D., C. Oelschlaeger, S. Müller, J. Küstner, S. Bindgen, and N. Willenbacher, 2014, Elongational deformation of wormlike micellar solutions, J. Rheol. 58, 20172042.

Thielicke, W. and E.J. Stamhuis, 2014, PIVlab Towards user friendly, affordable and accurate digital particle image veloci metry in MATLAB, J. Open Res. Softw. 2, e30.

Utracki, L.A. and J. Lara, 1984, Extensional flow of mica filled polyethylene, Polym. Compos. 5, 4451.

Weinberger, C.B. and J.D. Goddard, 1974, Extensional flow behavior of polymer solutions and particle suspensions in a spinning motion, Int. J. Multiph. Flow 1, 465486. 
Karlsruher Institut für Technologie

\section{Repository KITopen}

Dies ist ein Postprint/begutachtetes Manuskript.

Empfohlene Zitierung:

Oswald, W.; Recktenwald, S. M.; Willenbacher, N.

What controls filament thinning in uniaxial extension?

2019. Korea-Australia rheology journal, 31.

doi: $\underline{10.5445 / I R / 1000100833}$

Zitierung der Originalveröffentlichung:

Oswald, W.; Recktenwald, S. M.; Willenbacher, N.

What controls filament thinning in uniaxial extension?

2019. Korea-Australia rheology journal, 31 (4), 195-201.

doi:10.1007/s13367-019-0020-7 